\title{
Cross-tail current evolution during substorm dipolarization
}

\author{
A. T. Y. Lui \\ Johns Hopkins University Applied Physics Laboratory, Laurel, MD 20723-6099, USA \\ Correspondence to: A. T. Y. Lui (tony.lui@jhuapl.edu)
}

Received: 7 March 2013 - Revised: 7 May 2013 - Accepted: 24 May 2013 - Published: 27 June 2013

\begin{abstract}
We examine evolution of the cross-tail current during substorm current disruption/dipolarization using observations from two satellites in the near-Earth magnetotail at the downtail distances of $8-9 R_{\mathrm{E}}$. By choosing times when these two satellites are separated, mainly in the north-south distance in the tail current sheet, precise determination of current density in the layer embedded between these satellites can be obtained with Ampère's law. Two such events are examined and several common features are found. The current densities in the layer embedded by the two satellites were reduced by $\sim 40-70 \%$ during substorm dipolarization. The changes in current densities have the fast kinetic timescale, i.e., in seconds, implying a kinetic process for current disruption/dipolarization. The estimated power within the current layer was mainly dissipative in the dawn-dusk direction and mainly dynamo in the Sun-tail direction that is needed to drive the north-south substorm current system in the ionosphere. Remote sensing of the energization site with the ion sounding technique shows that the energization site was initially earthward of the satellite and moved down the tail at later times. Breakdown of the frozen-in condition occurred intermittently during the disturbance interval. These features provide important clues to the substorm onset process.
\end{abstract}

Keywords. Magnetospheric physics (Storms and substorms)

\section{Introduction}

A space plasma phenomenon that exhibits impulsive energy releases is the space disturbance known as a substorm. This disturbance was originally conceived from evolution of auroral displays in the polar region (Akasofu, 1964). It is now viewed as a prototype of many impulsive events in the plasma universe. A major feature of substorms on Earth is called "dipolarization", in which the magnetic field configuration in the nightside of the magnetosphere changes from a sheet-like magnetic field geometry to one resembling a dipole. The former arises from the development of strong cross-tail current and the change in dipolarization can be explained in terms of a reduction of the cross-tail current during a substorm. It is generally accepted that this current reduction is linked to the development of a westward electrojet in the ionosphere during a substorm through the diversion of the cross-tail current in the magnetosphere to the ionosphere.

There are two major paradigms proposed for substorms, namely, near-Earth current disruption and magnetic reconnection. The former paradigm envisions one or more plasma instabilities in the typical radial distance of $\sim 6-12 R_{\mathrm{E}}$ in the near-Earth region to be activated to cause a substorm onset. The initial disturbance subsequently triggers an avalanche of instability excitation spreading down the tail in multiple sites. The latter paradigm envisions the onset of magnetic reconnection at the typical radial distance of $\sim 15-30 R_{\mathrm{E}}$ in the midtail region. This activity gives rise to fast plasma flows towards Earth, leading subsequently to near-Earth dipolarization and substorm onset. Since the initial disturbance location is distinctly different in these paradigms, a NASA mission called Time History of Events and Macroscale Interactions during Substorms (THEMIS) was launched on 17 February 2007 with the primary goal of resolving the controversy between these two paradigms by placing five identically-instrumented satellites along the tail axis to determine incontrovertibly the propagation direction of substorm disturbances in the magnetotail (Angelopoulos, 2008). The apogees of these five satellites line up once every 4 days. Three inner probes have apogees at $\sim 10 R_{\mathrm{E}}$ while two outer ones have apogees at $\sim 20$ and $\sim 30 R_{\mathrm{E}}$.

In a recent article, Lui (2011) examined magnetic field observations of a substorm interval from two THEMIS satellites in the near-Earth region (radial distance of $\sim 8 R_{\mathrm{E}}$ ) separated 


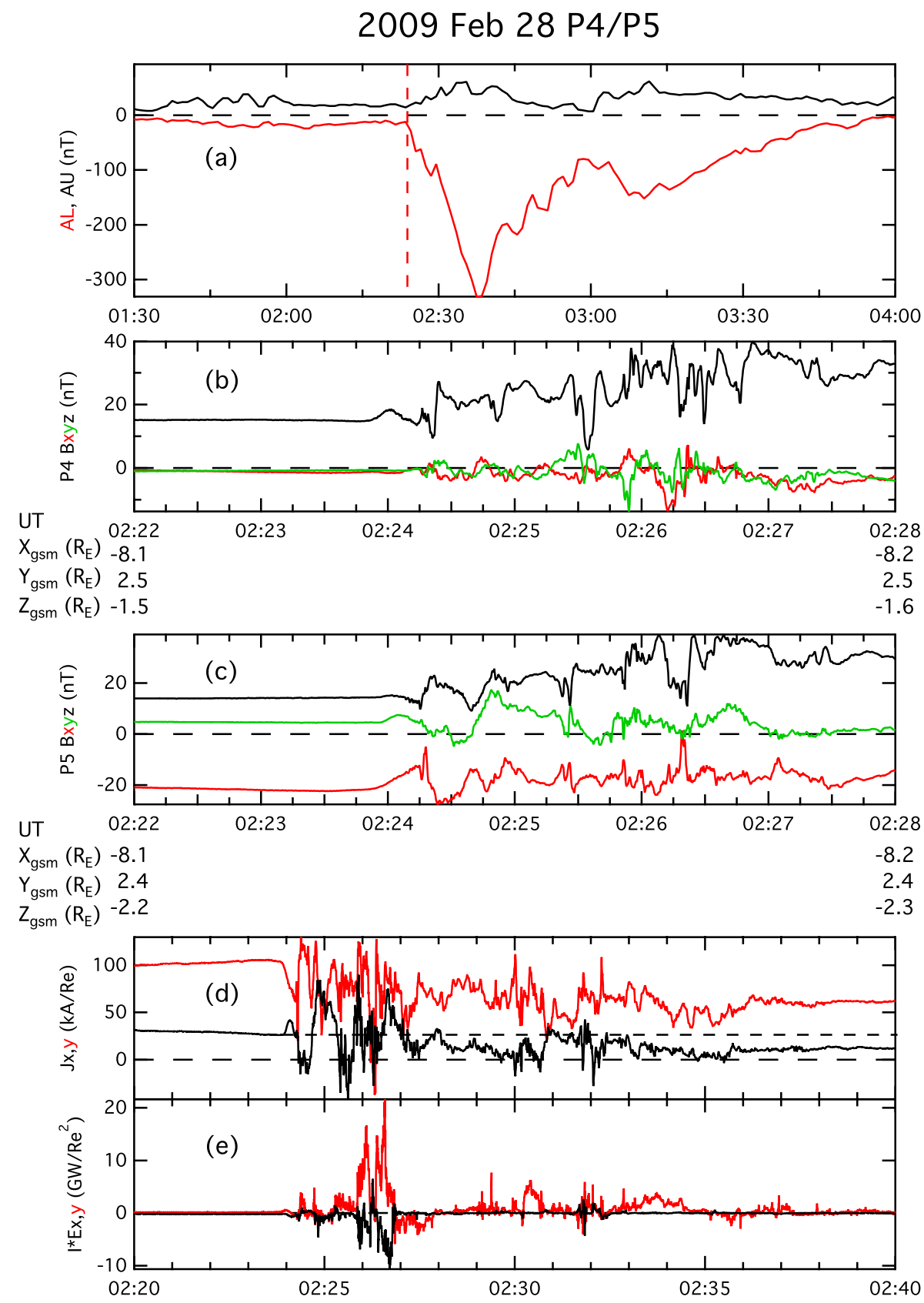

Fig. 1. Observations of the current disruption/dipolarization event on 28 February 2009: (a) the AU/AL indices; (b) and (c) the magnetic field components in GSM coordinates at P4 and P5 (different colors are used for different components, as indicated by the label), and current densities in the layer embedded by P4 and P5; and (d) and (e) power calculated from the product of current densities and the electric field averaged over the two satellite measurements.

in the north-south direction by $\sim 0.7 R_{\mathrm{E}}$ and with nearly identical projections on the equatorial plane. The substorm onset is indicated by both ground-based magnetic activity from magnetic stations and auroral activity from all-skycamera station. The current densities in the near-Earth region during the current disruption/dipolarization (CDD) interval were found to vary in a timescale of seconds. The current densities were eventually reduced by $\sim 40-45 \%$ within the layer embedded by the two satellites. Furthermore, it was inferred that the particle energization site associated with CDD approached the satellite from the earthward-dawnward direction at the neutral sheet based on the observed anisotropy of energetic ions with the application of the ion sounding technique. In other words, the substorm onset location was earthward of $\sim 8 R_{\mathrm{E}}$ in the near-Earth region, consistent with the current disruption substorm paradigm. 
In this study, we first provide additional confirmation that the observed anisotropy of energetic ions in the event reported by Lui (2011) is genuinely due to remote sensing of energization site and not due to plasma flow. The amount of dissipation/dynamo power in that CDD event is also presented. Another CDD event from THEMIS observations is presented here to show that the results obtained earlier are not unique to just one event.

\section{Observations}

THEMIS data used in this study are magnetic field from the fluxgate magnetometer (FGM) (Auster et al., 2008), electric field from the electric field instrument (EFI) (Bonnell et al., 2008), plasma parameters and distribution functions from the Electrostatic Analyzer (ESA) (McFadden et al., 2008), and energetic particle fluxes from the Solid State Telescope (SST) (Angelopoulos, 2008). FGM measures magnetic field and its low frequency fluctuation up to $64 \mathrm{~Hz}$. EFI provides waveform and spectral three-axis measurements of the ambient electric field from DC up to $8 \mathrm{kHz}$, with a single, integral broadband channel extending up to $400 \mathrm{kHz}$. ESA measures three-dimensional distribution function from a few $\mathrm{eV}$ up to $26 \mathrm{keV}$ for electrons and up to $20 \mathrm{keV}$ for ions in $3 \mathrm{~s}$ spin period. SST obtains three-dimensional distribution function from $35 \mathrm{keV}$ to $6 \mathrm{MeV}$ for ions and from $30 \mathrm{keV}$ to $6 \mathrm{MeV}$ for electrons in $3 \mathrm{~s}$ spin period.

\subsection{February 2009 event}

A near-Earth CDD event on 28 February 2009 was examined in Lui (2011). Figure 1 shows some salient features of this event. A substorm onset indicated by the AU/AL indices occurred at 02:24 UT. The substorm was a moderate size one judging from the minimum AL value of $-331 \mathrm{nT}$ for this activity. This AL activity onset is collaborated well with the auroral breakup seen at 02:24 UT by the all-sky-camera at KUUJ station (see Fig. 6 in Lui, 2011). The two THEMIS satellites, P4 and P5 both at $X_{\mathrm{gsm}}=-8.1 R_{\mathrm{E}}$, had nearly identical equatorial projections but separated by the distance from the neutral sheet. Their north-south separation was $\sim 0.7 R_{\mathrm{E}}$. Before CDD onset at 02:23:49 UT, both $B_{\mathrm{X}}$ and $B_{\mathrm{y}}$ components at $\mathrm{P} 4$ had small values $\left(\left|B_{\mathrm{x}}\right|,\left|B_{\mathrm{y}}\right|<1.5 \mathrm{nT}\right)$ in comparison with the $B_{\mathrm{Z}}$ component, indicating the close proximity of $\mathrm{P} 4$ to the neutral sheet. $\mathrm{CDD}$ onset started with a small increase in the $B_{\mathrm{Z}}$ component, followed by large fluctuations with a high value of $39.7 \mathrm{nT}$ and a low value of $6.4 \mathrm{nT}$ during the subsequent $4 \mathrm{~min}$. At 02:28 UT, its value became $32.8 \mathrm{nT}$. The $B_{\mathrm{Z}}$ variations and the subsequent increase are characteristics of CDD near the neutral sheet.

P5 was further away from the neutral sheet than P4, as indicated by the high magnitude of the $B_{\mathrm{X}}$ component. However, considerable fluctuations in the $B_{\mathrm{Z}}$ component were detected. Since P4 and P5 had nearly identical equatorial pro- jections, the current densities embedded in the layer between these two satellites can be obtained with the application of the integral form of Ampère's law. Magnetic field data sampled with a time resolution of $0.25 \mathrm{~s}$ were used in this calculation. The changes in current densities over a larger time interval (02:20-02:40 UT) are shown in Fig. 1d. It is found that $J_{\mathrm{y}}$ increased slightly from $\sim 100$ to $106 \mathrm{kA} / R_{\mathrm{E}}$ just prior to the CDD onset, while $J_{\mathrm{x}}$ decreased slightly from $\sim 32$ to $26 \mathrm{kA} / R_{\mathrm{E}}$. At CDD onset, there was a sharp drop in $J_{\mathrm{y}}$ down to $61 \mathrm{kA} / R_{\mathrm{E}}$ accompanied by a substantial increase in $J_{\mathrm{x}}$ up to $42 \mathrm{kA} / R_{\mathrm{E}}$, suggesting a part of $J_{\mathrm{y}}$ was directed earthward initially. After onset, both $J_{\mathrm{x}}$ and $J_{\mathrm{y}}$ varied tremendously, with occasional values larger than their values before onset. The timescale of variations was very short, i.e., in the kinetic regime of seconds. For example, at 02:24:46UT, $J_{\mathrm{y}}$ changed from 126 to $85 \mathrm{kA} / R_{\mathrm{E}}$ in $3 \mathrm{~s}$. At 02:26:18 UT, $J_{\mathrm{y}}$ changed from -17 to $119 \mathrm{kA} / R_{\mathrm{E}}$ in $3 \mathrm{~s}$. Similar rapid changes were seen in $J_{\mathrm{x}}$. At 02:24:38 UT, $J_{\mathrm{x}}$ changed from 2 to $34 \mathrm{kA} / R_{\mathrm{E}}$ in $3 \mathrm{~s}$. At 02:25:58 UT, $J_{\mathrm{x}}$ changed from 42 to $4 \mathrm{kA} / R_{\mathrm{E}}$ in $3 \mathrm{~s}$. The value of $J_{\mathrm{x}}$ even became negative at several short intervals. At the end of the interval (02:40 UT), $J_{\mathrm{y}}$ and $J_{\mathrm{x}}$ settled down to 62 and $12 \mathrm{kA} / R_{\mathrm{E}}$, respectively. These values represent reductions of $\sim 42 \%$ and $\sim 54 \%$.

It is important to distinguish the substorm current system from the R1/R2 current system generated by the solar windmagnetosphere dynamo (Iijima and Potemra, 1976). In the premidnight sector, $J_{\mathrm{X}}$ for R1/R2 near the neutral sheet is positive, as shown by the observed value prior to the CDD onset. Therefore, to isolate $J_{\mathrm{x}}$ current associated with the substorm, the current value prior to CDD onset needs to be used as the base line, which is indicated by the dotted line in Fig. $1 \mathrm{~d}$ and has the value of $26 \mathrm{kA} / R_{\mathrm{E}}$. With this base line, one can see that $J_{\mathrm{x}}$ for the substorm current system due to CDD was often directed tailward. At the end of this interval, it became $12 \mathrm{kA} / R_{\mathrm{E}}$, well below its value prior to CDD onset. The averaged value of $J_{\mathrm{x}}$ in the interval 02:23:4902:40:00 UT is $16 \mathrm{kA} / R_{\mathrm{E}}$, implying an averaged tailward directed current of $10 \mathrm{kA} / R_{\mathrm{E}}$ near the neutral sheet for the north-south substorm current system in the ionosphere.

The amount of power involved in the CDD can be estimated by forming the dot product of the current density with the electric field averaged over the two satellites. For accurate electric field measurements, the power calculation was done in the despun spacecarft coordinates, which is close to the GSE coordinations. Positive values of power implies dissipation while negative value implies dynamo action. In the $\mathrm{y}$-direction, the power was mainly dissipation although there were brief intervals with a weak dynamo effect. In the xdirection, the power was mainly negative, i.e., a dynamo.

Near the neutral sheet, there are two possibilities in causing particle anisotropy for an isotropic population in its own rest frame of reference: one is due to plasma flow and the other is due to remote sensing of particles within the distance of the particle's gyroradius. The second alternative can be due to pressure gradient with a well-defined magnetic 

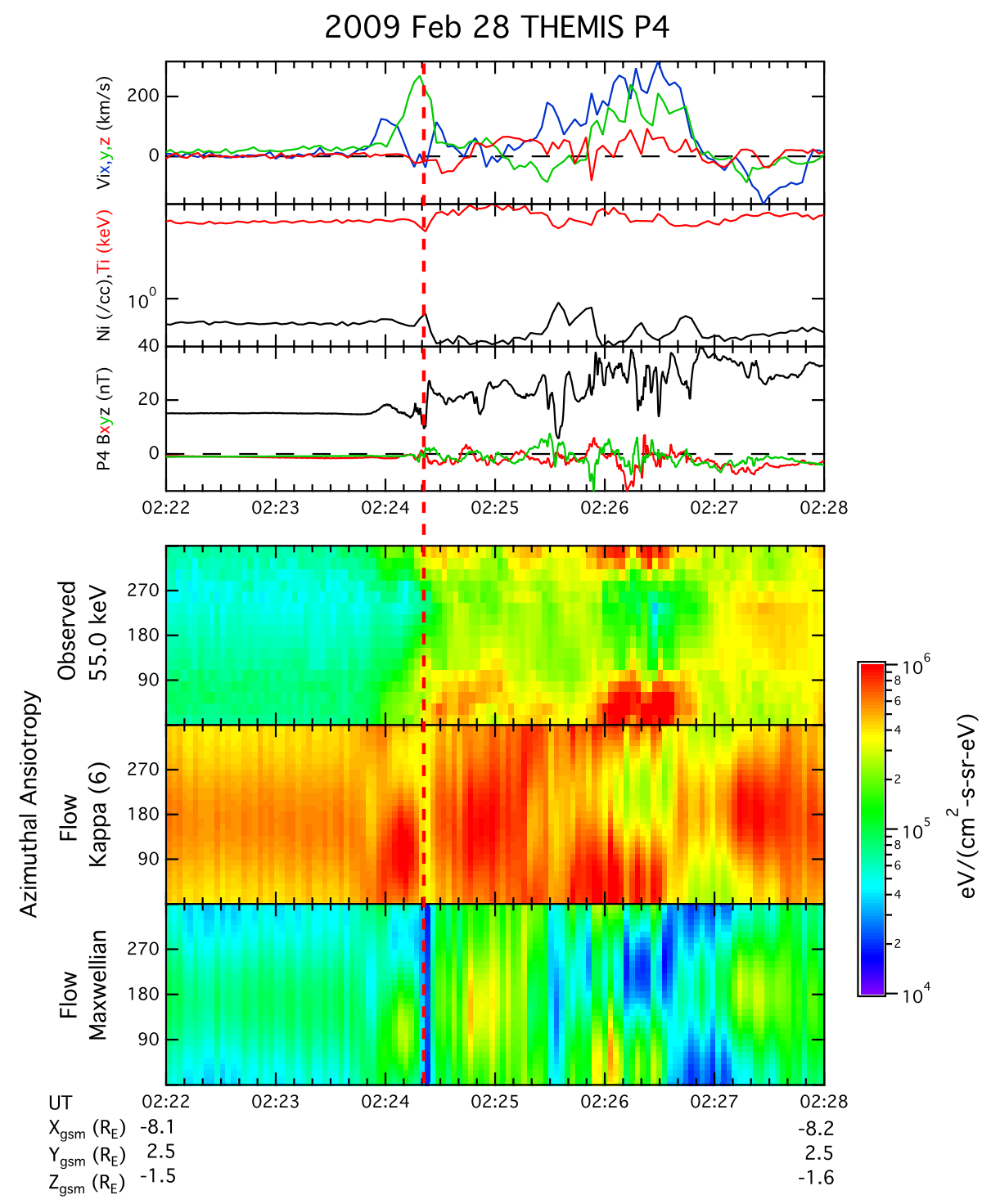

Fig. 2. Observations of current disruption/dipolarization event on 28 February 2009 from THEMIS P4. From the top to bottom are the plasma flow components in GSM coordinates (different colors are used for different components, as indicated by the label), the number density and temperature of ions, the magnetic field components in GSM coordinates (different colors are used for different components, as indicated by the label), the observed azimuthal anisotropy of $50-60 \mathrm{keV}$ ions, the anisotropy expected from plasma flow with a kappa distribution of $\kappa=6$, and the anisotropy expected from plasma flow with a Maxwellian distribution.

structure or energization site showing its evolution such as in CDD events. In other words, if the observed anisotropy is not due to plasma flow and shows a significant temporal evolution, then the anisotropy of energetic ions observed near the neutral sheet can be used to infer the location of the particle energization site associated with CDD relative to the satellite P4. In order to demonstrate this anisotropy is not due to plasma flow, we have calculated the expected anisotropy of energetic ions from plasma flow and compared results with the observed anisotropy. The expected flow anisotropy for $55 \mathrm{keV}$ ions due to plasma flow is calculated for a population with kappa distribution of $\kappa=6$ and one with a
Maxwellian distribution (corresponding to $\kappa=$ infinity) using the observed number density, velocity, and temperature. The plasma is assumed to be isotropic in its rest frame. For a kappa distribution with a bulk flow $\overline{\boldsymbol{u}}$, the differential intensity of particles with velocity $\overline{\boldsymbol{v}}$ in any direction can be calculated by the following expression:

$j(\overline{\boldsymbol{v}})=\frac{N v^{2}}{m w^{3 / 2}} \frac{\Gamma(\kappa+1)}{\kappa^{3 / 2} \Gamma(\kappa-1 / 2)}\left[1+\frac{(\overline{\boldsymbol{v}}-\overline{\boldsymbol{u}})^{2}}{\kappa w^{2}}\right]^{-(\kappa+1)}$,

where $N$ is the number density, $m$ is the particle mass, $w$ is the thermal speed, and $\Gamma$ denotes the gamma function. Note 


\section{Feb 28 P4 55 keV lons 022414-022500 UT}
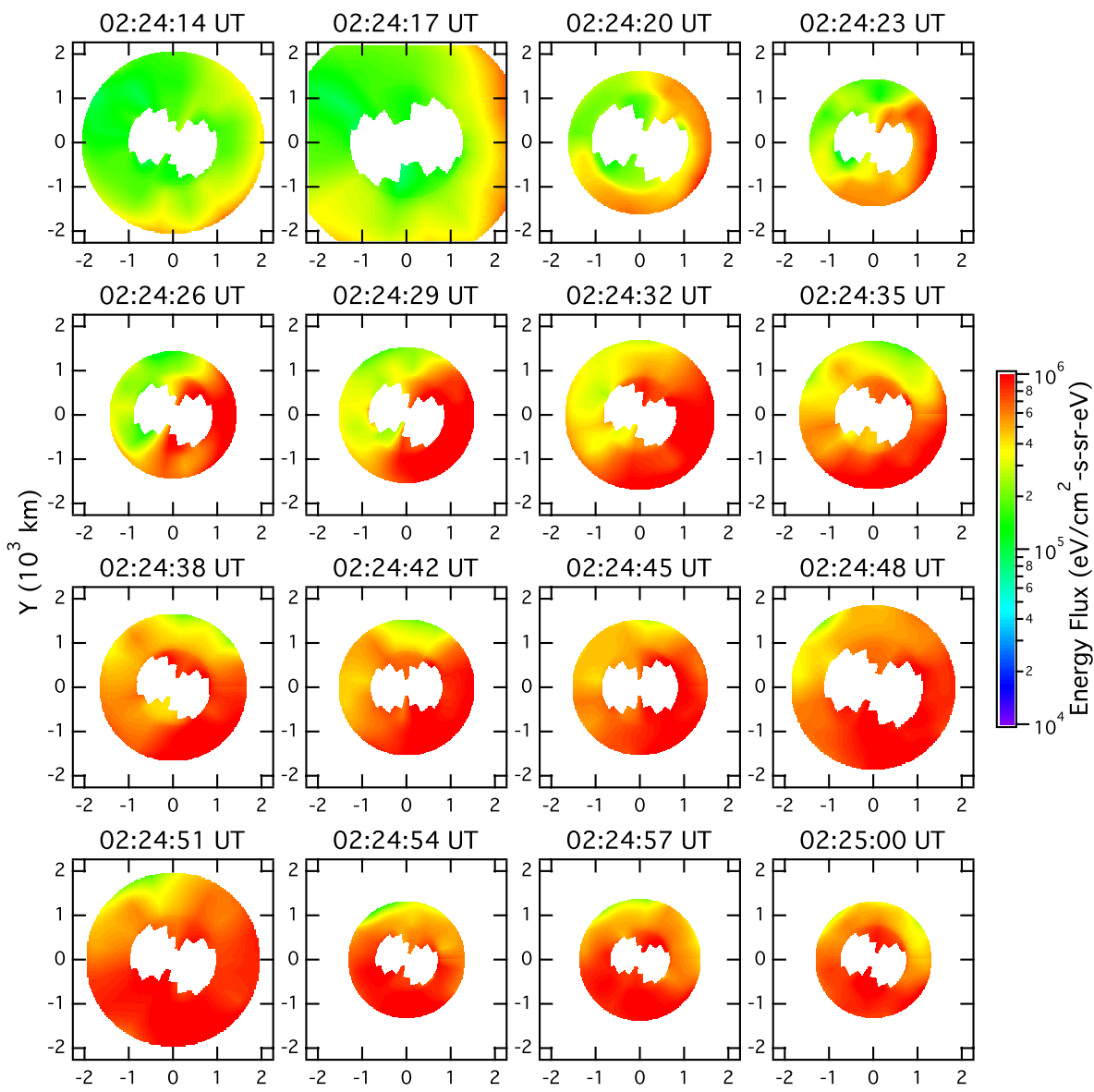

\section{然}

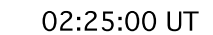

$$
\mathrm{X}\left(10^{3} \mathrm{~km}\right)
$$

Fig. 3. Time evolution of energy fluxes for $50-60 \mathrm{keV}$ ions around the satellite THEMIS P4, constructed by mapping the measured energy fluxes to the particle's gyrocenters.

that $\overline{\boldsymbol{v}}$ and $\overline{\boldsymbol{u}}$ are vectors and the square of their difference is sum of squares of their differences in three components. For a Maxwellian distribution, the corresponding differential intensity is given by

$$
j(\overline{\boldsymbol{v}})=\frac{N v^{2}}{m \pi^{3 / 2} w^{3}} \exp \left[-\frac{(\overline{\boldsymbol{v}}-\overline{\boldsymbol{u}})^{2}}{w^{2}}\right] .
$$

The result is shown in Fig. 2. Since the observed fluxes generally lie between the expected fluxes from the kappa and Maxwellian populations, the observed fluxes are consistent with a population having a kappa value larger than 6 .

The vertical line marks the onset time 02:24:20 UT for significant energization and dipolarization for this event. For $\sim 30 \mathrm{~s}$ prior to this onset time, significant anisotropy was observed in association with the duskward flow of up to $\sim 270 \mathrm{~km} \mathrm{~s}^{-1}$. Plasma flow components became small right after the onset time. However, the observed anisotropy was enhanced significantly, contrary to the expected decrease in anisotropy if it were due to plasma flow, as seen in the panels showing the anticipated anisotropy. This anticorrelation and the appearance of a time evolving structure confirm that the observed anisotropy right after the onset time is due to remote sensing of particle energization site.

The movement of the energization site can be revealed better by projecting the observed particles to their gyrocenters with respect to the satellite using the averaged magnetic field over the sampling interval. This is shown in Fig. 3. The larger distance covered in some of these plots reflects the smaller value of the averaged magnetic field in the sampling interval. At the beginning of this interval, the energization site was sensed at a distance of $\sim 2000 \mathrm{~km}$ directly earthward of the satellite at $X_{\mathrm{gsm}}=-8.1 R_{\mathrm{E}}$. This indicates that CDD had occurred earlier at a location earthward of the satellite. At a later time, e.g., 02:24:35 UT, it moved to be earthward-dawnward of the satellite. At 02:25:00 UT, it moved to tailward-dawnward of the satellite. It may be noted that the energization site did not move at a constant speed 


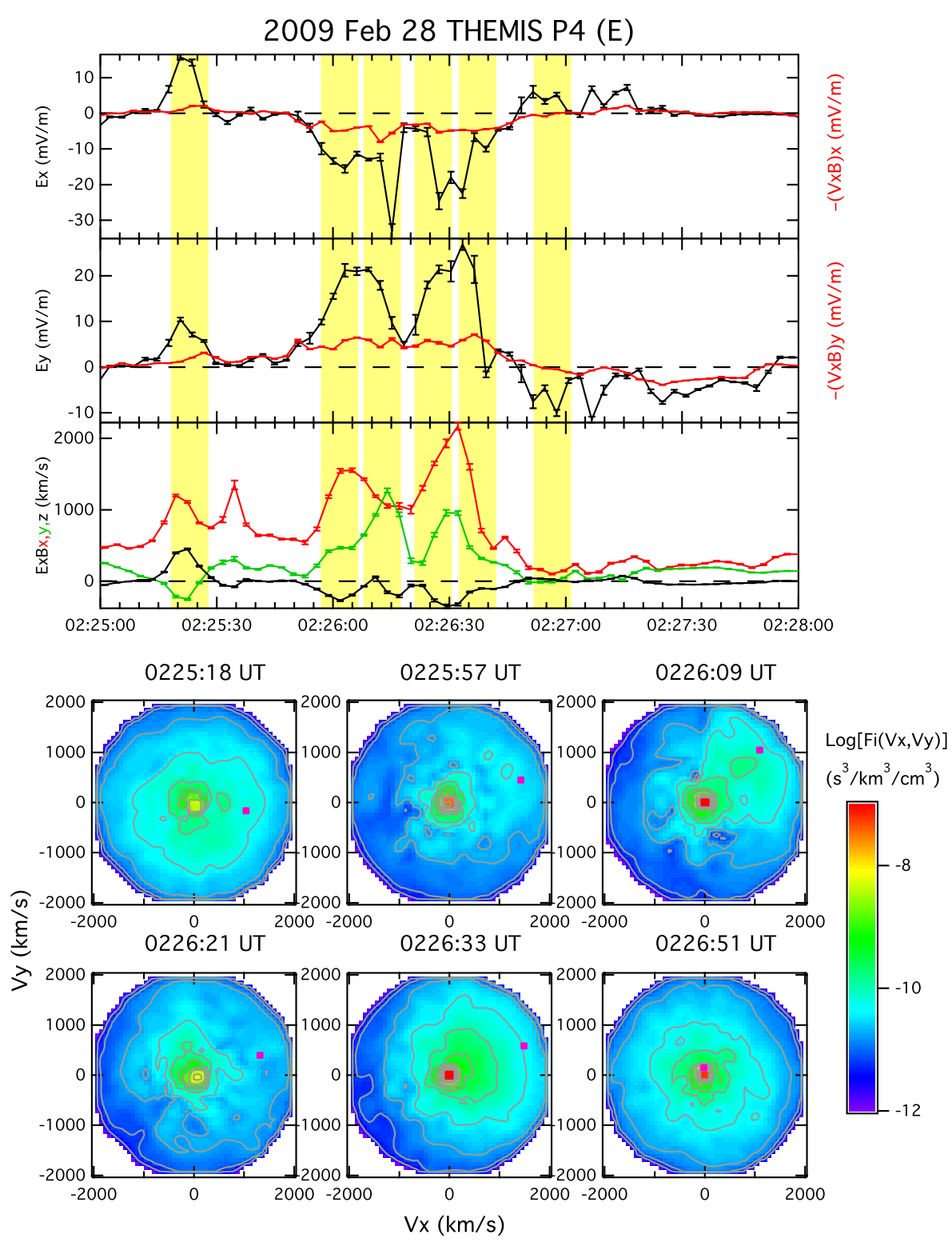

Fig. 4. Comparison between observed electric field components $E_{\mathrm{x}}$ and $E_{\mathrm{y}}$ and the values expected from the frozen-in condition of $E=$ $-\boldsymbol{V} \times \boldsymbol{B}$. The third panel shows the expected plasma flow components from the frozen-in condition. Error bars shown in the top three panels denote the standard errors of the mean. The bottom two rows show a sequence of the ion velocity distributions in the perpendicular flow components $V_{\mathrm{x}}$ and $V_{\mathrm{y}}$ for comparison to indicate the observed velocity distributions do not match the required velocity shifts (denoted by the red dot) needed to match the $\boldsymbol{E} \times \boldsymbol{B}$ plasma flows.

down the tail since tailward spreading of CDD onset is not related to any wave propagation in the near-Earth current disruption paradigm.

Figure 4 shows the occasional breakdown of the frozenin condition during CDD. The observed electric field components $E_{\mathrm{x}}$ and $E_{\mathrm{y}}$ in the despun spacecraft coordinates are compared with that inferred from the measurements of plasma flow $\boldsymbol{V}$ and magnetic field $\boldsymbol{B}$ based on the frozenin condition $E=-\boldsymbol{V} \times \boldsymbol{B}$ expected for plasma behaving as a fluid under the MHD condition. Highlighted are six in- tervals showing the departure of the observed electric field from the expected frozen-in electric field. Another check on the breakdown of the frozen-in condition is by comparing the $\boldsymbol{E} \times \boldsymbol{B}$ velocity with the observed ion velocity. The third panel shows the $\boldsymbol{E} \times \boldsymbol{B}$ velocity in despun satellite coordinates based on the frozen-in condition, i.e., using the observed electric and magnetic field with the assumption of $\boldsymbol{E} \times \boldsymbol{B}=0$. During this interval, the x-component of the $\boldsymbol{E} \times \boldsymbol{B}$ velocity reached above $2000 \mathrm{~km} \mathrm{~s}^{-1}$ while the y-component reached above $1200 \mathrm{kms}^{-1}$. The bottom two 
2009 Mar 05 P4/P5
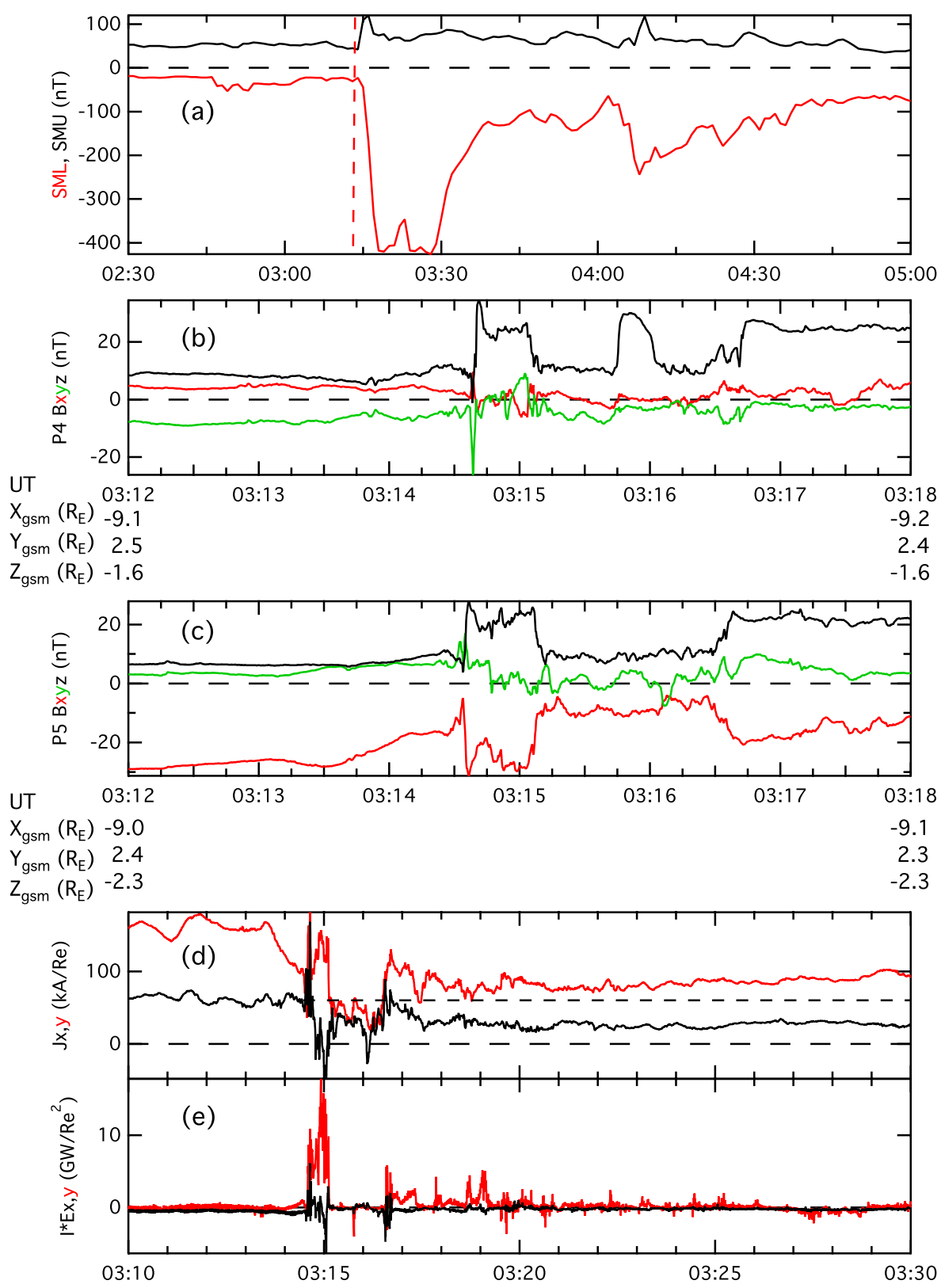

Fig. 5. Observations of the current disruption/dipolarization event on 5 March 2009. The parameters shown and the format are same as Fig. 1.

rows show the ion velocity distribution on the plane of perpendicular velocity components $V_{\mathrm{x}}$ and $V_{\mathrm{y}}$ corresponding to the highlighted time intervals. If one compares the velocity distribution at these times and the required $\boldsymbol{E} \times \boldsymbol{B}$ velocity to satisfy the frozen-in condition, indicated by the red dot in each panel, it is evident that the observed velocity distribution does not match the shift for the required $\boldsymbol{E} \times \boldsymbol{B}$ velocity. This further reinforces the result that frozen-in condition was broken occasionally during the CDD interval.

\subsection{March 2009 event}

Figure 5 shows the relevant parameters for another CDD event on 5 March 2009. The ground magnetic disturbances indicating substorm activity are provided by the SuperMAG magnetic indices SMU and SML (Gjerloev, 2009). These indices are similar to AU and AL, but SuperMAG network has far more magnetic stations than those used in the determination of $\mathrm{AU}$ and $\mathrm{AL}$. As a result, more coverage of ground magnetic disturbances can be obtained by SMU/SML than AU/AL. This substorm is also a moderate size one, with 

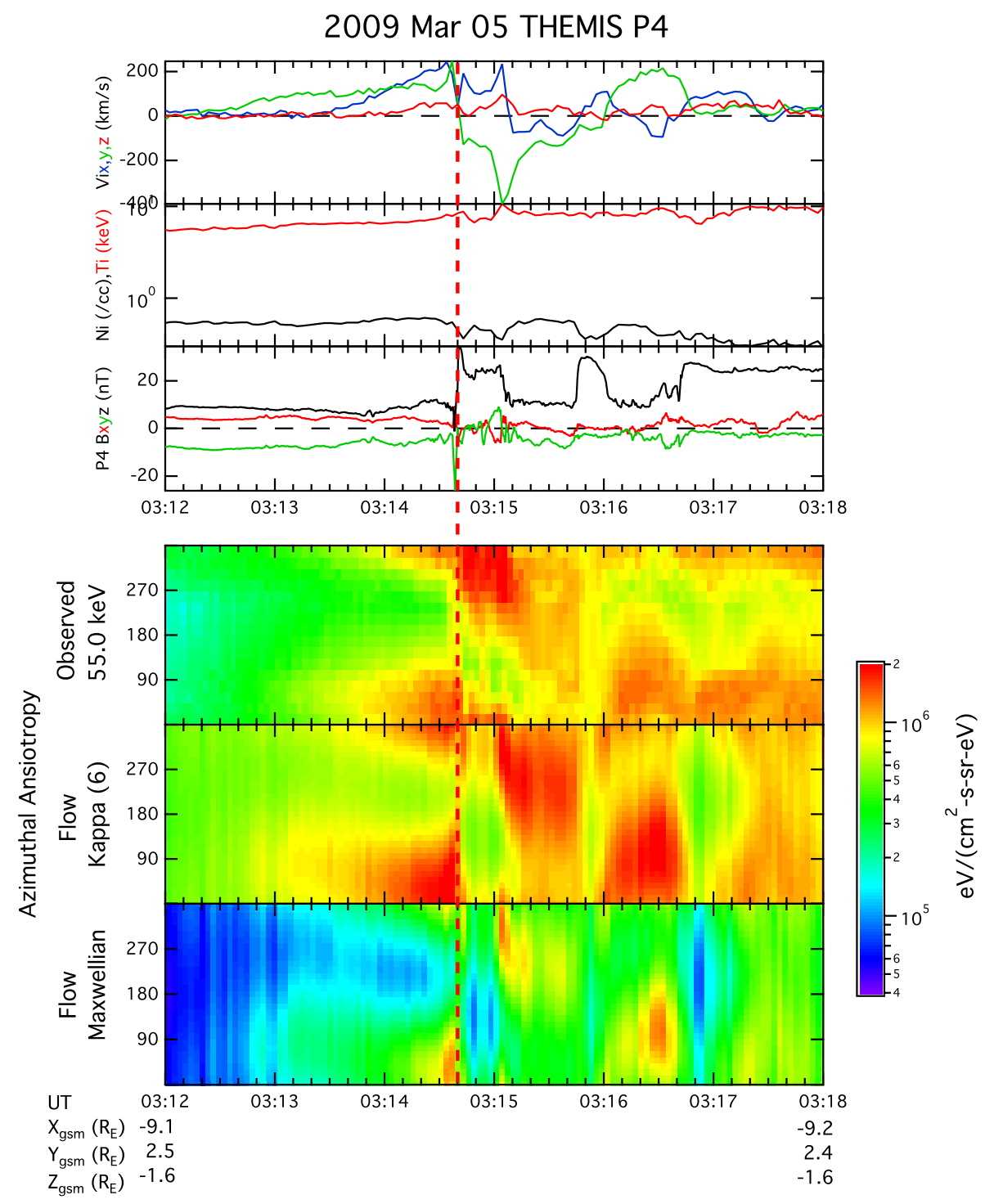

Fig. 6. Observations of the current disruption/dipolarization event on 5 March 2009. The parameters shown and the format are same as Fig. 2.

the first minimum SML at $-418 \mathrm{nT}$. The all-sky-cameras at SNKQ and KUUJ stations also showed auroral brightening starting at 03:12 UT, followed by a significant poleward expansion at 03:14 UT.

For this event, both $\mathrm{P} 4$ and P5 had nearly the same equatorial projections and at $X_{\mathrm{gsm}} \approx-9 R_{\mathrm{E}}$. The two satellites were separated by $\sim 0.7 R_{\mathrm{E}}$ in the z-direction. $\mathrm{P} 4$ was closer to the neutral sheet than P5, as indicated by the magnitude of the $B_{\mathrm{x}}$ component detected by both satellites. The first indication of current reduction was noted at P5 when the $B_{\mathrm{X}}$ component showed a gradual but significant decrease for $\sim 1$ min long, accompanied by a gradual increase in $B_{\mathrm{Z}}$ component. About the same time, there was also a noticeable increase of $B_{\mathrm{Z}}$ component at P4. A sharp increase of the $B_{\mathrm{Z}}$ component at both satellites occurred later after $\sim$ 03:14 UT but lasted for
$<1 \mathrm{~min}$. Sustained dipolarization started at 03:16:30 UT and 03:16:25 UT for P5 and P4, respectively.

A larger time interval (03:10-03:30 UT) is used to compute the current density and power. Using Ampère's law on magnetic field data at $0.25 \mathrm{~s}$ time resolution, we find the reduction of $J_{\mathrm{y}}$ starting at $\sim$ 03:13:30 UT, likely a result of current disruption at some distance from the satellite location. Near the onset of abrupt dipolarization (03:14:36 UT), $J_{\mathrm{y}}$ decreased briefly from $\sim 163$ to $103 \mathrm{kA} / R_{\mathrm{E}}$ in $6 \mathrm{~s}$, accompanied by a brief increase of $J_{\mathrm{X}}$ from $\sim 38$ to $66 \mathrm{kA} / R_{\mathrm{E}}$ within the corresponding time interval. This behavior is similar to the previous event, suggesting a slight redirection of the crosstail current to earthward-directed current at the initial stage of CDD. At later times, $J_{\mathrm{y}}$ had a brief recovery but eventually showed a significant decrease compared with its initial value before the activity. For $J_{\mathrm{x}}$, after its brief increases, it 


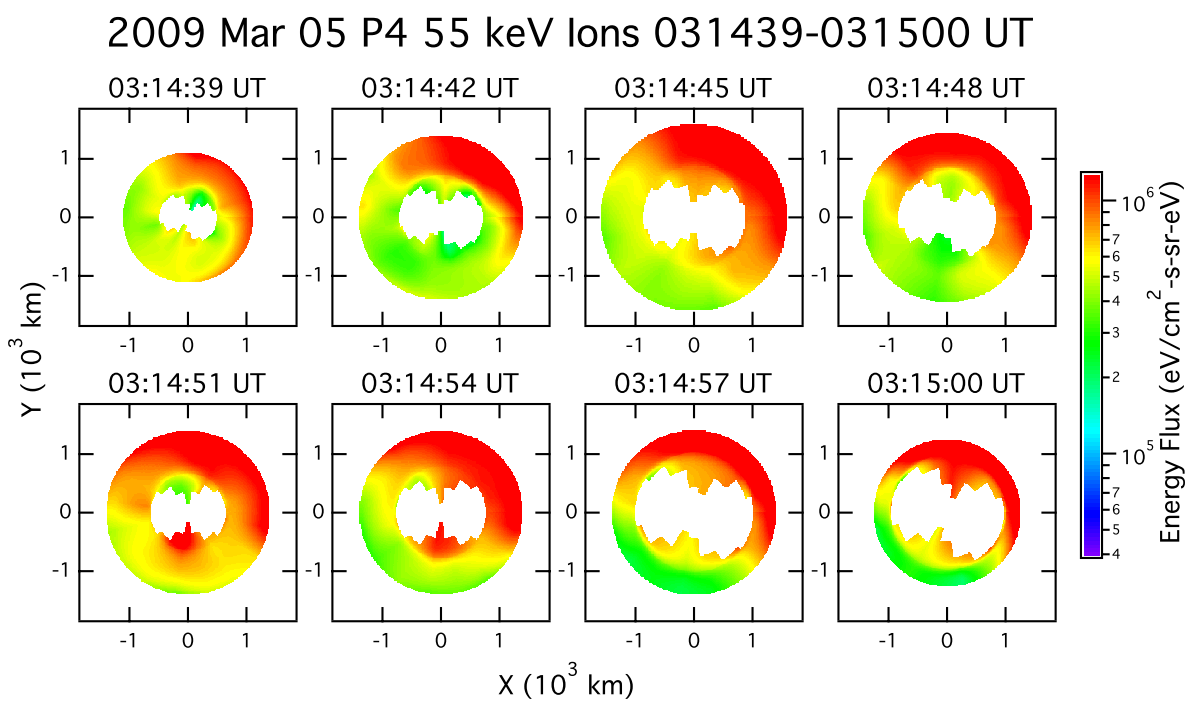

Fig. 7. Remote sensing of energization site for the current disruption/dipolarization event on 5 March 2009, with format same as Fig. 3.

began to decrease significantly, even reversing in direction briefly before settling down to a positive value. At 03:22 UT, $J_{\mathrm{y}}$ and $J_{\mathrm{x}}$ had values of 73 and $19 \mathrm{kA} / R_{\mathrm{E}}$, representing decreases of $\sim 55 \%$ and $\sim 68 \%$ from their initial values, respectively. Similar to the previous event, the timescale of current changes is in seconds. As before, to isolate $J_{\mathrm{x}}$ current associated with $\mathrm{R} 1 / \mathrm{R} 2$ current system, a base line of $60 \mathrm{kA} / R_{\mathrm{E}}$ is shown in Fig. $5 \mathrm{~d}$ to visualize the $J_{\mathrm{x}}$ change associated with the substorm current system due to CDD. With this base line, it can be seen that $J_{\mathrm{x}}$ was earthward initially for a brief moment (like the first event) but was almost always tailward afterwards. The average value of $J_{\mathrm{x}}$ in the time interval 03:14:20-03:30:00 UT is $28 \mathrm{kA} / R_{\mathrm{E}}$, implying an averaged tailward directed current of $32 \mathrm{kA} / R_{\mathrm{E}}$ near the neutral sheet for the north-south substorm current system in the ionosphere.

We also estimate the power/dynamo for this event from the dot product of the current density with the electric field averaged over the two satellites. The result shows that it was mainly dissipative in the $y$-direction. In the $\mathrm{x}$-direction, the power was mainly negative, i.e., a dynamo. This is also similar to the result for the previous event.

Figure 6 shows the evaluation of whether the observed anisotropy of energetic ions arises from plasma flow or from remote sensing of the CDD energization site. When the energy fluxes in the three anisotropy panels are compared, it can be inferred that the ion population had a kappa value larger than 6 since the observed energy fluxes were between the fluxes shown in the two calculated anisotropy. Prior to the abrupt dipolarization at P4, there was a substantial earthward-duskward anisotropy that can be related to plasma flow. However, at the time of dipolarization onset, the observed fluxes in the $270-360^{\circ}$ angular sector far exceeded that expected from flow anisotropy for $\sim 1.5 \mathrm{~min}$. Therefore, the observed anisotropy associated with significant time variation is a result of remote sensing of the energization site.

Figure 7 shows the sequence of projections created by mapping the observed fluxes to their respective gyrocenters. It can be seen that the energization site was initially earthward-duskward of the satellite. At a later time, it moved down the tail to a position mostly duskward of the satellite. The motion was not uniform though.

Figure 8 shows that the frozen-in condition was broken intermittently during this CDD interval. During this breakdown in the highlighted time interval, the top two panels show that the observed electric field components were larger than the values inferred from $E=-\boldsymbol{V} \times \boldsymbol{B}$. Furthermore, the inferred ion velocities based on the frozen-in condition (marked by red dots in the velocity distribution plots) were also much higher than the velocity shift in the ion velocity distributions during the breakdown interval. The results of these two checks are similar to those found in the previous event.

\section{Summary and discussion}

We have examined in detail two events of near-Earth CDD observed by two THEMIS satellites located at the downtail distances between 8 and $9 R_{\mathrm{E}}$. The CDD onset occurred near substorm onset, as indicated by the ground magnetic disturbances and by the auroral activity from the corresponding all-sky-camera stations. During the CDD interval, the two satellites had nearly identical equatorial projections and their separation in the z-direction was only $\sim 0.7 R_{\mathrm{E}}$. This configuration offers the opportunity to determine the current densities embedded within the layer between the two satellites from the Ampère's law. Several interesting features common to these two events are the following: 


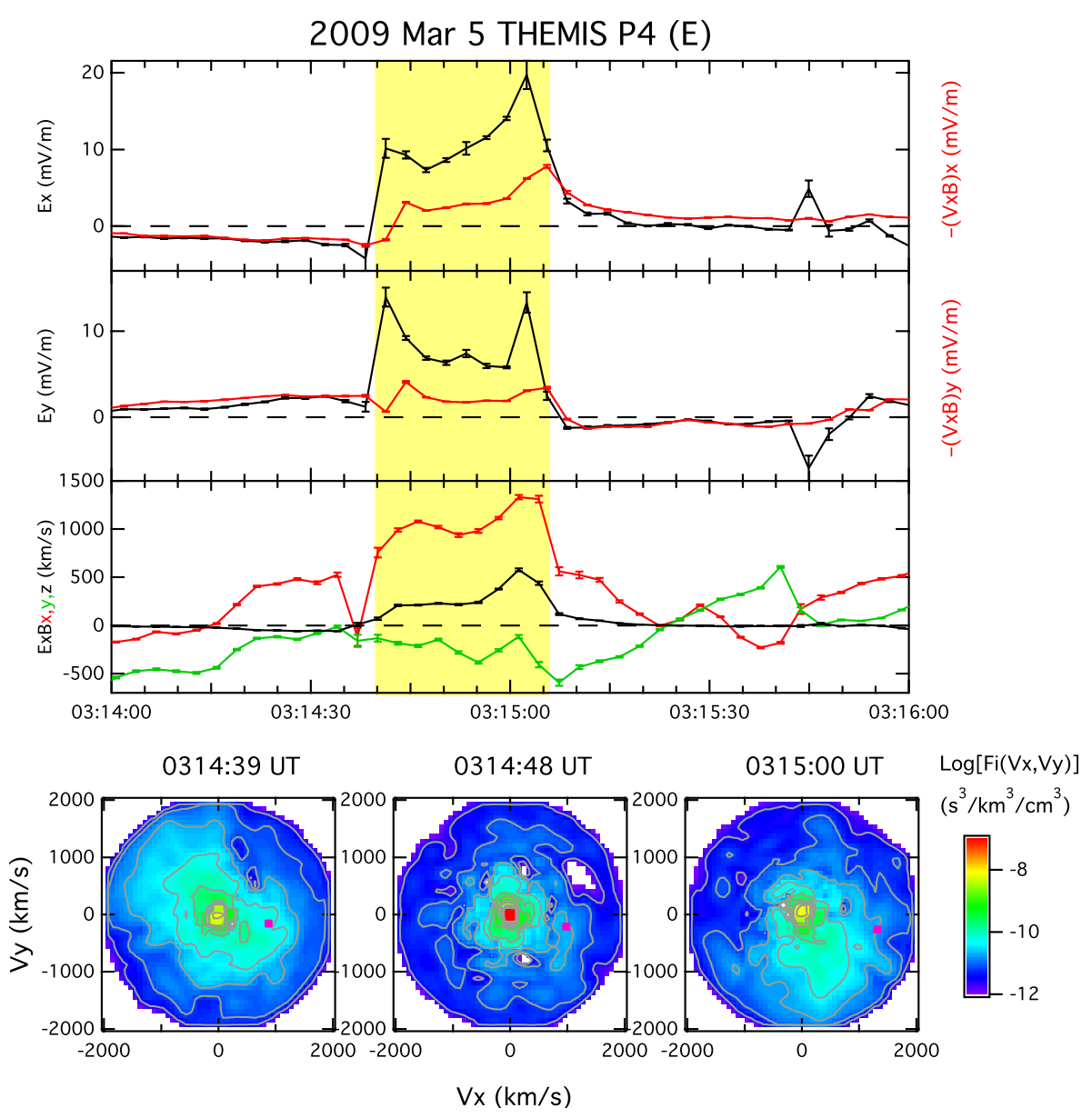

Fig. 8. Observations for the current disruption/dipolarization event on 5 March 2009. The parameters shown and the format are same as Fig. 4.

1. The changes in the current densities embedded in the layer between the two satellites during CDD were significant, with the eventual current reduction in the neighborhood of $\sim 40-70 \%$ from initial values of $\sim$ $100-170 \mathrm{kA} / R_{\mathrm{E}}$ before CDD onset.

2. The current densities fluctuated considerably with a timescale in the kinetic regime of seconds.

3. There was a temporal increase in the $J_{\mathrm{X}}$ component accompanying the initial decrease in the $J_{\mathrm{y}}$ component.

4. By isolating the $J_{\mathrm{x}}$ contribution from $\mathrm{R} 1 / \mathrm{R} 2$ current system, the $J_{\mathrm{x}}$ component due to CDD was found to be almost always directed tailward.

5. With electric field measurements, the associated power/dynamo was estimated to be in tens of $\mathrm{GW} / R_{\mathrm{E}}^{2}$.

6. The power in the dawn-dusk direction was typically positive, i.e., dissipation, while the power in the suntail direction was typically negative, i.e., dynamo.
7. Associated with the CDD onset was a short interval during which the anisotropy of energetic ions observed by the satellite close to the neutral sheet was due to remote sensing of CDD energization site and not due to plasma flow.

8. The initial location of the CDD energization site was earthward of the satellite location, i.e., earthward of the downtail distances of $8-9 R_{\mathrm{E}}$.

9. The CDD energization site was observed to move downtail at later times.

10. Occasional breakdown of the frozen-in condition was found during CDD in which the observed electric field components far exceeded the values inferred from $E=$ $-\boldsymbol{V} \times \boldsymbol{B}$. The velocity shifts of the observed ion velocity distributions were much less than inferred from $V=\boldsymbol{E} \times \boldsymbol{B} / \boldsymbol{B}^{2}$.

These findings provide important clues about the physical process for CDD and substorm onset. The short timescale 
suggests a kinetic process such as the cross-field current instability (Lui et al., 1991; Yoon and Lui, 1993) and short time-scale form of ballooning instability (Liu et al., 2012). These rapid variations are expected since CDD has been shown to have characteristics of turbulence, as demonstrated by fractal and multifractal analyses (Ohtani et al., 1995; Lui and Najmi, 1997; Lui, 2002) as well as scaling features of the probability distribution function (Consolini et al., 2005). The fact that both $J_{\mathrm{x}}$ and $J_{\mathrm{y}}$ fluctuated considerably suggests the breakup of the cross-tail current into filamentary currents within the CDD site, as shown by particle simulation of the cross-field current instability (Lui, 2004). These are the fine structures in the substorm current system, including its associated field-aligned current based on the current continuity condition. They are likely to be related to the fine structures in auroras seen during substorm activity.

The initial increase in $J_{\mathrm{x}}$ with decrease in $J_{\mathrm{y}}$, coupled with the evidence of CDD starting earlier earthward of the satellite, suggests that current redirection in the x-direction may be an indication of partial replenishment of the current disrupted at the earthward site.

The location of CDD inferred from remote sensing of energetic ions indicates that substorm onset process in the tail can occur in a region where the magnetic field normal to the neutral sheet can be very strong, ruling out the possibility of magnetic reconnection as the substorm onset process. Furthermore, in these two events, CDD was associated with dissipation in the dawn-dusk direction, an indication of energy conversion from field to particles. It was also associated with a dynamo in the sun-tail direction, an indication of the driver location near the neutral sheet for the north-south current portion of the substorm current system in the ionosphere as depicted in Lui and Kamide (2003). In their scenario, the kinetic plasma instability is triggered when the ions become unmagnetized. During CDD, electrons move earthward with the collapsing field lines while the ions cannot keep up with the electron motion (the field line slippage condition). This produces an earthward pointing electric field and a tailward directed current simultaneously, i.e., creating a dynamo in this process.

One may note that fast transient current changes in CDD events have shorter durations than the entire dipolarization interval seen locally at the satellite. This difference in timescale is expected from the substorm onset scenario proposed in the near-Earth current disruption paradigm, e.g., Lui et al. (1991). The kinetic plasma instability has global consequences since it reduces the local current density, changes the local Lorentz force to upset the prior force balance, and causes a collapse of the stretched field line configuration. In addition, it induces plasma sheet thinning in adjacent regions by sending a rarefaction wave downtail. This can instigate the onset of the kinetic instability at a slightly tailward site if sufficient plasma sheet thinning is achieved later to excite the instability. The further collapse of the stretched field line configuration in this adjacent thin plasma sheet can lead to a more sustained dipolarization earthward of this adjacent site. This situation is similar to an avalanche in which a small local disturbance can lead to a large-scale one with totally different time and spatial scales from the local disturbance.

It should be noted that since there is a time delay between onset of plasma sheet thinning due to arrival of the rarefaction wave and time interval required for the plasma sheet to thin down enough to excite the current disruption instability, the speed for tailward spreading of current disruption cannot be equated to the rarefaction wave speed.

Acknowledgements. This work was supported by the NSF grant ATM-0852508 and NASA grant NNX12AP62G to The Johns Hopkins University Applied Physics Laboratory, and by NAS502099 for THEMIS mission support. We acknowledge NASA contract NAS5-02099 and V. Angelopoulos for use of data from the THEMIS mission through AIDA at Institute of Space Science, National Central University in Taiwan; C. W. Carlson and J. P. McFadden for use of ESA data; D. Larson and R. P. Lin for use of SST data; J. W. Bonnell for use of EFI data; and K.-H. Glassmeier, U. Auster and W. Baumjohann for use of FGM data provided under the lead of the Technical University of Braunschweig and with financial support through the German Ministry for Economy and Technology and the German Center for Aviation and Space (DLR) under contract 50 OC 0302.

Guest Editor M. Balikhin thanks N. Ganushkina and one anonymous referee for their help in evaluating this paper.

\section{References}

Akasofu, S.-I.: The development of the auroral substorm, Planet. Space Sci., 12, 273-282, 1964.

Angelopoulos, V.: The THEMIS mission, Space Sci. Rev., 141, 453-476, doi:10.1007/s11214-008-9336-1, 2008.

Auster, H. U., Glassmeier, K. H., Magnes, W., Aydogar, O., Baumjohann, W., Constatntinescu, D., Fischer, D., Fornacon, K. H., Georgescu, E., Harvey, P., Hillenmaier, O., Kroth, R., Ludlam, M., Narita, Y., Nakamura, R., Okrafka, K., Plaschke, E., Richer, I., Schwarzl, H., Stoll, B., Valavanoglou, A., and Wiedemann, M.: The THEMIS fluxgate magnetometer, Space Sci. Rev., 141, 235-264, 2008.

Bonnell, J. W., Mozer, F. S., Delory, G. T., Hull, A. J., Ergun, R. E., Cully, C. M., Angelopoulos, V., and Harvey, P. R.: The electric field instrument (EFI) for THEMIS, Space Sci. Rev., 141, $303-$ 341, doi:10.1007/s11214-008-9469-2, 2008.

Consolini, G., Kretzschmar, M., Lui, A. T. Y., Zimbardo, G., and Macek, W. M.: On the magnetic field fluctuations during magnetospheric tail current disruption: A statistical approach, J. Geophys. Res., 110, A07202, doi:10.1029/2004JA010947, 2005.

Gjerloev, J. W.: A Global Ground-Based Magnetometer Initiative, EOS, 90, 230-231, 2009.

Iijima, T. and Potemra, T. A.: The amplitude distribution of fieldaligned currents at northern high latitudes observed by Triad, J. Geophys. Res., 81, 2165-2174, 1976.

Liu, W. W., Liang, J., Donovan, E. F., and Spanswick, E.: If substorm onset triggers tail reconnection, what trig- 
gers substorm onset?, J. Geophys. Res., 117, A11220, doi:10.1029/2012JA018161, 2012.

Lui, A. T. Y.: Multiscale phenomena in the near-Earth magnetosphere, J. Atmos. Solar-Terr. Phys., 64, 125-143, 2002.

Lui, A. T. Y.: Potential plasma instabilities for substorm expansion onset, Space Sci. Rev., 113, 127-206, 2004.

Lui, A. T. Y.: Reduction of the cross-tail current during near-Earth dipolarization with multi-satellite observations, J. Geophys. Res., 116, A12239, doi:10.1029/2011JA17107, 2011.

Lui, A. T. Y. and Kamide, Y.: A fresh perspective of the substorm current system and its dynamo, Geophys. Res. Lett., 30, 1958, doi:10.1029/2003GL017835, 2003.

Lui, A. T. Y. and Najmi, A.-H.: Time-frequency decomposition of signals in a current disruption event, Geophys. Res. Lett., 24, 3157-3160, 1997.
Lui, A. T. Y., Chang, C.-L., Mankofsky, A., Wong, H.-K., and Winske, D.: A cross-field current instability for substorm expansions, J. Geophys. Res., 96, 11389-11401, 1991.

McFadden, J. P., Carlson, C. W., Larson, D., Angelopoulos, V., Ludlam, M., Abiad, R., Elliott, B., Turin, P., and Marckwordt, M.: The THEMIS ESA plasma instrument and in-flight calibration, Space Sci. Rev., 141, 277-302, doi:10.1007/s11214-008-9440-2, 2008.

Ohtani, S., Higuchi, T., Lui, A. T. Y., and Takahashi, K.: Magnetic fluctuations associated with tail current disruption: fractal analysis, J. Geophys. Res., 100, 19135-19145, 1995.

Yoon, P. H. and Lui, A. T. Y.: Nonlinear analysis of generalized cross-field current instability, Phys. Fluids B, 5, 836-853, 1993. 